\title{
Immune-related pathophysiological causes relevant to a subset of patients with preeclampsia (Review)
}

\author{
HIROSHI KOBAYASHI, MAYUKO ICHIKAWA, JURIA AKASAKA, TAIHEI TSUNEMI and TOSHIYUKI SADO \\ Department of Obstetrics and Gynecology, Nara Medical University, Kashihara, Nara 634-8522, Japan
}

Received March 18, 2019; Accepted April 16, 2019

DOI: $10.3892 /$ wasj.2019.10

\begin{abstract}
Preeclampsia is characterized by inadequate extravillous trophoblast (EVT) invasion, leading to feto-placental hypoxia, a release of an excessof anti-angiogenic factors, and finally eliciting maternal endothelial dysfunction and maternal symptoms, such as new-onset hypertension and proteinuria. Despite intensive research over the past decade, the initial pathogenesis of this disorder remains elusive. There are likely different subtypes of preeclampsia and maternal, fetal and placental factors all play an important role in the disease development. In this review, we focus on an immune-related pathway or pathophysiological causes relevant to a subset of patients with preeclampsia. Using the PubMed database, we conducted a literature review of various studies related to the pathogenesis of preeclampsia. The two-stage theory is a notable postulate of the disease. The antecedents of poor placentation are immunological, epigenetic and environmental factors in origin. The causes of inadequate immune mechanisms at the maternal-fetal interface in preeclampsia is considered to be a lack of human leucocyte antigen (HLA) class I molecules, such as HLA-G and HLA-C. A reduction in HLA molecules facilitate EVTs to elicit an immune attack that furthers cell killing. A series of the HLA-G promoter region on EVTs has been found to be more highly methylated in preeclampsia than in normal pregnancy, possibly due to the increased expression of DNA methyltransferase-1 (DNMT-1). Promoter hypermethylation is one of the major epigenetic alterations that may prime for HLA-G gene inactivation. Epigenetic alterations are reversible, and nutrition, lifestyle and environmental factors may be the epigenetic regulators that modify gene expression. However, the timing, cause and underlying mechanisms of HLA gene methylation have not yet been fully established. The adverse intrauterine environment that contributes to immune responses, inflammation, or oxidative stress may be associated
\end{abstract}

Correspondence to: Professor Hiroshi Kobayashi, Department of Obstetrics and Gynecology, Nara Medical University, 840 Shijo-cho, Kashihara, Nara 634-8522, Japan

E-mail: hirokoba@naramed-u.ac.jp

Key words: preeclampsia, extravillous trophoblast cells, human leucocyte antigen, epigenesis, environment with increased susceptibilities for a number of adult diseases, including preeclampsia. We have hypothesized that close interactions between the inherited epigenetic architecture (hypermethylation of HLA molecules) and adverse intrauterine environmental exposures (oxidative stress and inflammation) leading to epigenetic modifications and to the aberrant DNA methylation of HLA class I molecules, may act as an early event of preeclampsia development.

\section{Contents}

1. Introduction

2. Data collection methods

3. Gene expression profiling and functional pathway analysis of preeclampsia

4. Immune cells as the triggers of preeclampsia development

5. HLA gene expression on EVTs in preeclampsia

6. Epigenetic regulation of HLA genes in preeclampsia

7. Conclusions and considerations

\section{Introduction}

Preeclampsia is a pregnancy complication characterized by new-onset hypertension and proteinuria that may be accompanied by generalized maternal endothelial dysfunction and end organ dysfunction. This disease leads to maternal and perinatal morbidity and mortality, and it also confers an increased long-term risk of chronic illnesses, including preterm birth, babies with low birth weight, cardiovascular disease and stroke (1-4). Emerging evidence suggests that the etiopathogenesis is multifactorial, resulting from a combined contribution of dietary and demographic factors, maternal genetic predisposition, epigenetic alterations and environmental insults (4-7). There are likely different subtypes of preeclampsia, which may arise from different etiopathogenic backgrounds, and maternal, fetal and placental factors all play a role (8). Early-onset preeclampsia is likely caused by placental dysfunction. Late-onset preeclampsia develops predominantly due to the metabolic mismatch and disturbances, including obesity and diabetes. Both diseases affect endothelial function.

A number of researchers thus far have speculated that the placenta may be the main pathogenetic focus of preeclampsia, as the disease-related symptoms end with the delivery of 
the placenta (9). Extravillous trophoblast (EVT) invasion and spiral artery remodeling are abnormal in women who develop preeclampsia, which leads to decreased placental perfusion and hypoxia (9-11). Poor placentation leads to the secretion of factors related to processes, such as oxidative stress, anti-angiogenesis and aberrant maternal systemic inflammation, into the maternal circulation and then causes generalized maternal endothelial dysfunction characterized by increased vasoconstriction, hypertension, and other manifestations of organ dysfunction $(9,12-16)$. Taken together, the two-stage model is a pathophysiological cause relevant to a subset of patients with preeclampsia and a notable postulate of the disease in which the abnormal spiral arteries modification leads to a poorly perfused placenta (stage 1) and produces factors, leading to the clinical manifestations of preeclampsia (stage 2) (17). Vascular dysfunction is presented as a downstream effect of substances released by a poorly perfused placenta. Although the initial cause of preeclampsia remains unknown, abnormal EVT invasion appears to play an important role in the pathogenesis of preeclampsia.

Furthermore, emerging evidence indicates that preeclampsia has stage-specific gene signatures: Immune escape from natural killer (NK) cell attack may enhance EVT invasion ('stop' preeclampsia development) and inflammation, oxidative stress and an imbalance in the angiogenic factors promote disease progression ('go' preeclampsia development) $(4,7,13,14,18-20)$. This model underscores a stage-dependent 'go or stop' dichotomy that exerts opposing influences, possibly through a better ability to flexibly adapt to a changing environment. However, it would be of interest to determine what causes the abnormal EVT invasion and spiral artery modification. Previous studies have reported the alterations in the function of immune cells in relation to the degree of EVT invasion and increased susceptibility to preeclampsia, emphasizing the role of NK cells as an initial step of the pathogenesis of preeclampsia and mechanical progress (21-27). In this review, we choose to focus on an immune-related pathway or pathophysiological causes relevant to a subset of patients with preeclampsia.

\section{Data collection methods}

The authors are members of the Japan Society of Perinatal and Neonatal Medicine and have written at least three published articles about preeclampsia. Some members of this article were assigned to design and review the fields of the research. A computerized literature search was conducted to identify relevant studies reported in the English language. We collected a comprehensive literature search from the PubMed and Embase databases up to December 2018, combining the keywords 'preeclampsia, 'extravillous trophoblast cells', 'human leucocyte antigen (HLA)', 'killer cell immunoglobulin-like receptor (KIR)', 'genotype', 'pathogenesis', 'oxidative stress', 'epigenesis, 'inflammation', and 'environment'. A variety of combinations of these terms were used, depending on which database was searched. Furthermore, the references of each article were searched to identify potentially relevant studies. Publications of original studies and review articles were included, while those documenting opinions, points of view or anecdotes were discarded. The final draft was submitted following multiple revisions.

\section{Gene expression profiling and functional pathway analysis of preeclampsia}

The integrative 'omics' research approaches, specifically microarrays studies, are widely used to identify the potential biomarkers and to understand the pathogenesis of preeclampsia (28-31). With the emerging advancement of next-generation sequencing technologies, high-throughput RNA sequencing (RNA-seq) also provides a valuable resource of spatial-temporal gene expression patterns (32-34). Compared to microarray-based analysis, RNA-seq can detect splicing isoforms and somatic mutations by taking advantage of a higher coverage and greater resolution (34). These approaches have identified the gene expression signatures and have predicted key pathways involved in preeclampsia-affected placentas (31,32,35-38). Although to date, at least to the best of our knowledge, there are relatively few available studies which have conducted gene expression analysis in preeclampsia-affected placentas using the RNA-seq method $(32,33)$, a number of differentially expressed genes and their pathways have been shown to have the same direction of change.

First, the differentially expressed genes, including leptin $(L E P)$, fms-related tyrosine kinase 1 (FLT1), inhibin subunit alpha (INHA), pappalysin 2 (PAPPA2), chorionic gonadotropin subunit beta $(C G B)$, luteinizing hormone beta polypeptide $(L H B)$, sialic acid binding Ig like lectin 6 (SIGLEC6) and corticotropin-releasing hormone $(\mathrm{CRH})$, may contribute to the altered trophoblast physiology in preeclampsia $(31,32,37,38)$. Low maternal serum levels of pappalysin 1 (PAPP-A), galectin 13 (placental protein 13; PP13) and insulin like growth factor 1 (IGF-I), and high levels of homocysteine, adrenomedullin A (ADMA), soluble endoglin (sEng), LEP, sFlt-1, inhibin A and activin A are predictive of emerging preeclampsia (39). A majority of studies focusing on preeclampsia have shared several overlapping genetic findings on defective decidualization $(35,36,40)$. Abnormal decidualization leads to a decreased EVT invasion and to the development of preeclampsia, which corroborates the concept of the endometrial antecedents of preeclampsia $(40,41)$.

Second, Gene Ontology enrichment analysis and functional ingenuity pathway analysis have been used to identify potential signaling pathways, suggesting that various physiological processes, such as chronic inflammation characterized by local or systemic oxidative stress $(12,16)$ and innate and adaptive immune system $(13,14)$, angiogenesis, hypoxia, apoptosis and placental development are implicated in the pathophysiology of preeclampsia (15). The following biological processes are also enriched in preeclampsia: Metabolism, cell cycle regulation, implantation, decidualization, cell-cell adhesion, the response to cAMP, the transforming growth factor (TGF)- $\beta$ signaling pathway, Wnt signaling pathway and the Hippo signaling pathway $(35,42-45)$. The abnormal spiral artery remodeling leads to a state of relative ischemia and to an increase in oxidative stress. The anti-angiogenic molecule, sFlt-1, produced by the trophoblast and the sFlt-1/placental growth factor (PIGF) ratio are recognized as diagnostic and prognostic biomarkers of preeclampsia $(9,10)$. Thus, an imbalance between angiogenesisand anti-angiogenesis-related molecules has emerged as an important regulator of the progression of preeclampsia. 
However, as previously demonstrated, this pathway is known to be active in some women with preeclampsia, but not in others; half of patients with preeclampsia were shown to have normal levels of these markers (46). Women with pregnancies complicated by preeclampsia exhibit chronic inflammation characterized by pro-inflammatory cytokines, oxidative stress (reactive oxygen species) and agonistic autoantibodies to the angiotensin II (Ang II) and type 1 receptor (AT1-AA) (12). It would be of interest to identify any possible triggers of these abnormal pathways in susceptible individuals. Some factors may trigger disease initiation. Among these, the immune system may lie upstream as one of the underlying mechanisms of reduced placental perfusion (19). An unbalanced regulation of immune responses may aggravate systemic inflammation, oxidative stress and endothelial dysfunction, which provides new insight into the role of immune pathway in disease initiation $(13,14,47)$.

\section{Immune cells as the triggers of preeclampsia development}

During placental establishment, fetal EVTs invade the maternal decidua, remodel uterine spiral arteries to create the blood supply and establish adequate nutrition (21). Although EVTs express paternally derived semialloantigens, the maternal immune system must maintain immune tolerance toward EVTs to avoid inflammation that can threaten reproductive success (18). Differentially distributed immune cells, including the forkhead box P3 (FOXP3)-expressing regulatory T (Treg) cell subset, dendritic cells, NK cells and macrophages are important for the adequate development of the placenta in early pregnancy (26). The dysregulation of immune cell function may explain the development of obstetric complications, such as infertility, early and recurrent pregnancy loss, fetal growth restriction, premature birth and preeclampsia $(18,20)$.

Tregs. Tregs have potent immunosuppressive properties and play an important role in the maintenance of feto-maternal immune tolerance during gestation (18). Tregs promote reproductive fitness and facilitate EVT invasion into the uterine wall during the first trimester (18). Therefore, insufficient numbers of Tregs or a low Treg activity have been widely associated with preeclampsia, a shift toward Th1 responses (17). Several noteworthy review articles have been published on this matter $(17,18,48)$

NK cells. NK cells are a small, but significant immunoregulatory $\mathrm{T}$ cell subset of human peripheral blood lymphocytes. Uterine NK ( $\mathrm{uNK}$ ) cells are distinct from peripheral blood NK cells and increase in number during early pregnancy (27). uNK cells produce the immunoregulatory cytokines, interleukin (IL)-10 and TGF- $\beta$, inhibit cytotoxic T cell induction and inflammatory $\mathrm{T}$ helper type 17 cells, promote the generation of indoleamine 2,3-dioxygenase (IDO)-producing monocytes and Tregs and induce the apoptosis of effector T cells $(49,50)$. These data suggest that uNK cells control EVT invasion and vascular remodeling during the period of placental formation and play important roles in the maintenance of pregnancy $(24,49,50)$. During gestation, NK cells are influenced by a combination of two sets of genes; the maternal KIR genotypes (the expression of various activating and inhibitory receptors on NK cells) (51) and the trophoblast (fetal and paternal) HLA allotypes, including $H L A-G(52)$, $-E(53)$ and $-C$ (22). Certain combinations of KIRs on uNK cells and HLA class I molecules on EVTs at the fetal-maternal interface support successful immune adaptation, thereby facilitating increased NK cell function, trophoblast invasion, uterine arterial remodeling, placentation, embryo implantation and fetal growth $(21,49,54)$. An excess production of activating KIRs relative to inhibitory KIRs plays an important role in a successful pregnancy. In other words, greater numbers of inhibitory rather than activating KIRs are found in women with defective placentation, e.g., preeclampsia, fetal growth restriction or recurrent spontaneous abortion.

\section{HLA gene expression on EVTs in preeclampsia}

$H L A-G$. EVTs lack the appropriate expression of HLA-A and HLA-B molecules, although they express the unique pattern of classic polymorphic HLA-C and the non-classic invariant HLA-E and HLA-G $(55,56)$. The HLA-G molecule acts as a major contributor to fetal-maternal immune tolerance induction in pregnancy, allowing EVT invasion into the maternal spiral arteries and a successful pregnancy (52). HLA-G has immunomodulatory properties, promoting a shift from Th1 towards a Th2 response and providing a protective effect for the outcome of pregnancy (57). IL-10 is an anti-inflammatory and immunosuppressive master regulator that protects the host from immune responses (58). IL-10 upregulates HLA-G expression, induces immune tolerance and downregulates immune responses by decreasing the levels of pro-inflammatory cytokines, such as IL-1 (59). Serum IL-10 levels have been shown to be diminished in women with preeclampsia compared to women with normal pregnancies (60). These data are in agreement with recent findings, showing a reduced expression of IL-10 (60) and HLA-G (61) in preeclamptic trophoblasts. One of the causes of inadequate immune mechanisms at the maternal-fetal interface in preeclampsia is considered to be a lack of the IL-10-dependent HLA-G signaling pathway (60). Other underlying causes of preeclampsia include genetic variants and single nucleotide polymorphisms (SNPs) within the HLA-G molecule $(57,62)$. Dynamic alterations in the promoter CpG methylation and/or genetic variations in the 3' untranslated region (3'UTR) polymorphisms of HLA-G gene may play key roles in the HLA-G regulation (please see section below entitled 'Epigenetic regulation of HLA genes in preeclampsia').

$H L A-E$. HLA-E is other major histocompatibility complex (MHC) class Ib molecule that is expressed on EVTs $(62,63)$. To date, at least to the best of our knowledge, there is only one available study demonstrating that the decreased expression of HLA-E mRNA may be involve in the pathogenesis of preeclampsia (53). Although HLA-E expression has been shown to be repressed by DNA methylation in breast cancer (64), we have no information with respect to DNA hypermethylation of HLA-E molecule in preeclampsia placenta.

$H L A-C$. HLA-C is the only polymorphic HLA molecule expressed by EVTs at the maternal-fetal interface (49). The activation of $\mathrm{uNK}$ cells by KIR binding to HLA-C molecule promotes trophoblast invasion (22). The combination of NK cells lacking activating KIRs with a fetal HLA-C on EVTs 
predicts a risk of preeclampsia (65). In other words, a reduced expression of HLA-C may contribute to the inhibition of activating KIRs, which possibly leads to a suppression of immune escape from NK attack and trophoblast invasion. Further studies are required to determine whether the expression of HLA-C on EVTs is reduced in women with preeclampsia when compared to women with normal pregnancies.

Genetic combinations between KIRs and HLA molecules in preeclampsia. uNK cells serve as immunoregulators and express broad and diverse receptors mediating both activating and inhibitory signals. KIR haplotypes form two distinctive groups: KIR A haplotype and KIR B haplotype (66). The KIR A haplotype has predominantly inhibitory receptors (KIR2DL1, KIR2DL3, KIR2DL4, KIR3DL1, KIR3DL2 and KIR3DL3), while the B haplotype has mainly activating receptors (KIR2DS1, KIR2DS2, KIR2DS3, KIR2DS5 and KIR3DS1) (66). Genetic combinations between the maternal KIR genes and their fetal ligands (HLA molecules) are highly variable in each pregnant woman (49). The expression of activating KIRs on uNK cells reduces NK cell responsiveness and contributes to a successful pregnancy (22). There is convincing evidence to indicate that the lack of activating KIRs can lower uNK cell activation and predispose pregnant women to preeclampsia, suggesting that a KIR genetic variation may influence the risk of developing preeclampsia $(21,49,54)$.

In addition, EVTs escape NK cell detection by upregulating HLA haplotypes G, E and C. Several studies have identified that HLA genes are globally dysregulated in preeclampsia $(53,57,60,62,67)$. A reduced expression of HLA-G in the placenta $(61,68)$ and soluble HLA-G protein in serum (69) are considered to be associated with the development of preeclampsia. The maternal HLA-G genotype, HLA-G $1597 \Delta \mathrm{C}$ null mutation, leads to an increased risk of developing preeclampsia (70). The levels of HLA gene expression in whole peripheral blood (HLA-A) and placenta (HLA-DRB1) have also been shown to be altered in patients who have later developed the disorder (67). Therefore, the development of preeclampsia probably depends on certain genetic combinations of KIRs and HLA molecules.

\section{Epigenetic regulation of HLA genes in preeclampsia}

It would be of interest to determine what causes the aberrations of the HLA gene expression in susceptible individuals. HLA-G is induced by various stress-inducible conditions, including interferon (IFN)- $\beta$, IFN- $\gamma$, TGF- $\beta$, heat shock, hypoxia, arsenite and IDO, which leads to immune escape $(65,71,72)$. Progesterone also upregulates the expression of HLA-G, E and -C (73,74). By contrast, HLA-C expression has been shown to be downregulated in human trophoblasts via the inhibition of nuclear factor (NF)- $\kappa \mathrm{B}$ activation (75). The NLRP2 gene is a member of the nucleotide-binding and leucine-rich repeat receptor $(N L R)$ family, containing an $\mathrm{N}$-terminal pyrin effector domain (PYD), a centrally-located nucleotide-binding and oligomerization domain (NACHT) and C-terminal leucine-rich repeats (LRR) (75). The PYD is necessary and sufficient for the suppression of the activity of the pro-inflammatory transcription factor, NF- $\mathrm{kB} . N L R P 2$ is a maternally imprinted gene and is highly expressed by EVTs, and functions as an anti-inflammatory factor (75). However, little is known about the regulators of the spatial and temporal expression of HLA-G, -E and -C genes on EVTs.

To date, a substantial number of epidemiological studies have been published, indicating that preeclampsia is associated with an increased risk of hypertension, cardiovascular and metabolic diseases in later life (1-4). Maternal preeclampsia is a major determinant of an offspring's risk of developing cardiovascular disease, stroke and mental disorders during later adult life (4). The adverse intrauterine environment factors, including immune responses, inflammation, or oxidative stress, play a key role in causing this disorder. Adverse environmental programming in the early periods of life is a phenomenon that affects metabolic, physiological and psychological functions throughout life (7). Epigenetic modifications permit the fetus to adapt the expression of specific genes exposed to the adverse environment and leads to an increased risk of developing chronic diseases in later life (6).

Epigenetics and environmental factors, such as inflammation and oxidative stress can influence DNA methylation-related plasticity (76). Dynamic alterations of DNA methylation and demethylation are an example of aberrant epigenetic marks. One example is that environmental factors, such as oxidative stress can influence the expression of $\mathrm{CpG}$ demethylase, ten-eleven translocation (TET), which recognizes a wide range of endogenous DNA methyltransferases (DNMTs) (77). The TET gene mediates the hypermethylation of matrix metalloproteinase-9 (MMP-9), which reduces EVT invasion in preeclampsia (77). Not only alterations in the promoter and enhancer $\mathrm{CpG}$ methylation/demethylation, but also functional polymorphisms in the 3'UTR, may be the critical mechanisms involved in the downregulation of HLA class I molecule expression (78). A series of the HLA-G promoter region on EVTs have been shown to be more highly methylated in preeclampsia than in normal pregnancy due to the increased expression of DNMT-1 (61). Promoter hypermethylation is one of the major epigenetic alterations that may prime for $H L A-G$ gene inactivation. Furthermore, microRNAs (miRNAs) bind conserved 3'UTRs of the $H L A-G$ gene to prevent their translation, and a SNP in the 3'UTR affects the binding of miRNAs, which may influence miRNA targeting and HLA-G mRNA stability (79). The upregulation of HLA class I molecules on EVTs is an important mechanism which aids in the escape from maternal immune attack (80), while reduced levels of HLA molecules facilitate EVTs to elicit an immune attack that furthers cell killing (81). We thus hypothesized that adverse intrauterine environmental exposures leading to epigenetic modifications and the aberrant DNA methylation of HLA class I molecules may act as an early event for the development of preeclampsia. However, the timing, causes and underlying mechanisms of HLA gene methylation as regards the adverse intrauterine environment have not yet been fully established. We cannot currently describe how an adverse environment influences the expression of DNMT-1 and how the DNA methylation of HLA molecules affects the development of preeclampsia.

\section{Conclusions and considerations}

In this review, we first reviewed the literature to reveal the underlying molecular mechanisms responsible for the 


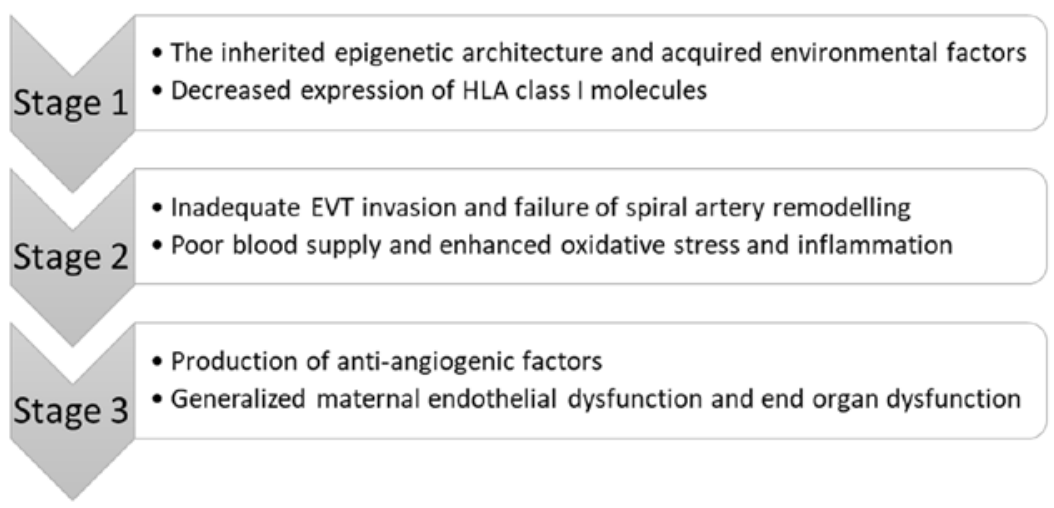

Figure 1. The three-stage model of preeclampsia. HLA, human leucocyte antigen; EVT, extravillous trophoblast.

development of preeclampsia.Previous studies have represented a comprehensive characterization of the preeclampsia transcriptome and have identified biomarkers and potential signaling pathways. The 'omics' studies, including microarray and RNA-seq approaches serve as attractive high-throughput, large-scale gene screening methods for the determination of differentially expressed genes. The majority of these transcriptome data and a network of signaling pathways have been obtained in placental tissue samples by case-control designed studies. The principal points of the present review are as follows: Preeclampsia is considered to be a disease of three stages (Fig. 1). The first stage concerns the decreased expression of HLA class I molecules, such as HLA-G and HLA-C genes, possibly through promoter hypermethylation or 3'UTR polymorphism of HLA molecules, leading to an inadequate interaction with $\mathrm{uNK}$ cell receptors, KIRs $(57,78,79)$. The HLA-related factors modulate the immune tolerance of the mother and allow EVTs to escape immune attack (80). A decreased HLA gene expression reduces the activity of antigen presentation, possibly enhancing the body's immune response against fetal trophoblasts (81). Alterations in the functions of immune cells, predominantly NK cells, in relation to the degree of EVT invasion, may play an initial role in etiopathogenesis of preeclampsia. However, the causes of the abnormal expression of HLA molecules should also be determined. The DNMT-1-mediated promoter hypermethylation of $H L A-G$ is associated with preeclampsia (61), suggesting that DNMT-1 upregulation may be an epigenetic marker in women who will develop preeclampsia later in life. Adverse intrauterine environmental factors, such as inflammation and oxidative stress may influence the expression of endogenous DNMTs or TET demethylase (77). Taken together, in the first stage theory, the inherited epigenetic architecture (hypermethylation of HLA molecules) and acquired environmental factors (oxidative stress and inflammation) may be an initial step to preeclampsia. Dysregulated placental immunity contributes to the next stage of preeclampsia (4). The second stage involves the inadequate EVT invasion that may result from the decreased expression of HLA class I molecules. A lack of EVT invasion results in poor placental perfusion. This process leads to the release of an excess of anti-angiogenic factors, which are responsible for generalized endothelial dysfunction and maternal symptoms, such as new-onset hypertension and proteinuria (19). The antecedents of poor placentation are considered to be immunological, epigenetic and environmental factors in origin $(5,22)$. The relative failure of early EVT invasion and the remodeling of the spiral arteries leads to a poor blood supply to the placenta, exposing it to oxidative stress and inflammation (9). The third stage is the maternal syndrome, which is characterized by a generalized systemic inflammatory response involving maternal endothelium through the production of pro-inflammatory cytokines and anti-angiogenic factors (19). Recent review articles have described the main role of anti-angiogenic factors in the pathogenesis of preeclampsia $(12,13)$. Maternal endothelial dysfunction can trigger maternal disease and can subsequently lead to clinical manifestations. The decreased HLA expression and increased immune attack may activate the domino-like effect of poor EVT invasion and then a generalized systemic inflammatory response.

Second, we addressed the immune-related pathways relevant to the initial causes of the development of preeclampsia. We discussed the interpretation and modeling of preeclampsia development, with a focus on the HLA molecules on EVTs as an initial stage of this disease. Recent evidence suggests that the interaction between KIRs on NK cells and HLA class I molecules on EVTs may be the point at which the apparent partner specificity of the disease originates. A number of studies have revealed significant differences in the numbers of individual activating and inhibitory KIR haplotypes genes between patients with preeclampsia and controls $(21,49,54)$. An excess production of inhibitory KIRs (KIR2DL1) relative to activating KIRs (2DS2, 2DS3 and 2DS5) has been shown to play an important role in the pathophysiology of defective placentation, e.g., preeclampsia or fetal growth restriction. The expression of HLA-G and HLA-C has been shown to be reduced in women with preeclampsia when compared to those with normal pregnancies (61). When the fetus possesses HLA-C, mothers who lack activating KIR have been shown to have an increased risk of developing preeclampsia. In other words, the reduced expression of HLA-G and HLA-C genes may possibly lead to the suppression of the immune escape from uNK attack (82). These findings strengthen the role of HLA function in the initial step to preeclampsia.

Third, prenatal adverse environments, such as maternal stress and toxicological exposures, can disrupt normal placental development and contribute to defective placentation (76). Although the placenta is the central organ for 
pathogenesis, epigenetic mechanisms in the placenta highlight the importance of a fine-tune orchestration of DNA methylation, miRNAs, histone modifications and chromatin structure (83). Recent epigenetic mechanisms associated with the dysfunction and dysregulation of decidualization-related genes have a critical impact on the susceptibility, development and progression of complex diseases, such as miscarriage, preterm birth, or preeclampsia $(35,36,40)$. Furthermore, a previous study demonstrated that half of the decidualization-related genes in preeclampsia-affected placentas are downregulated and are also located within and in close proximity to known imprinted genes, suggesting an association between an epigenetic process and genomic imprinting in preeclampsia (36). A wide variety of biological processes, including environmental factors, can modify the imprinting and heritable DNA methylation. The downregulated expression of HLA genes may be related to the higher levels of DNMT-1 transcripts, coding for marks that maintain the epigenetic status of cells (61). This suggests an epigenetic link between adverse intrauterine environments and the development of preeclampsia in later life.

Finally, this review includes two major weaknesses. There are likely different subtypes of preeclampsia (8). We wished to identify the initial cause of early-onset preeclampsia. Although the incidence of preeclampsia is increasing, early-onset disease is less common than late-onset. Hence, the number of patients with early-onset preeclampsia is likely to be limited in studies not specifically designed to examine this subgroup. Conclusions appear to be partially based on the assumption that studies conducted in women with all forms of preeclampsia apply to women with early-onset preeclampsia. Furthermore, this is not a systematic review. All findings are presented as equally certain, with no consideration of things, such as sample size, study design, blinded analysis of samples, or strengths and weaknesses of specific methodologies. These studies aid in our understanding of the (epi)genetic mechanisms underlying disease process; however, they are limited, as they do not come close to replicating the complexities that exist with in vitro and in vivo models.

In conclusion, our focus in this review was to demonstrate that improper immune responses are associated with the initial manifestation of the development of preeclampsia. Despite increasing evidence in the literatures addressing the role of genetics in the field of the pathogenesis of preeclampsia, very little is known about the epigenetic pathways involved in the initial step of this complex syndrome.

\section{Acknowledgements}

Not applicable.

\section{Funding}

The present study was supported by JSPS KAKENHI (grant no. 1398).

\section{Availability of data and materials}

Not applicable.

\section{Authors' contributions}

MI, JA and TT performed the literature search, collected the data regarding the epigenetic and genetic abnormalities and the underlying mechanism of preeclampsia development using the database. HK and TS made substantial contributions to the conception of the study. HK contributed to the study design and interpretation of the included research studies. The final version of the manuscript has been read and approved by all authors.

\section{Ethics approval and consent to participate}

Not applicable.

\section{Patient consent for publication}

Not applicable.

\section{Competing interests}

The authors declare that they have no competing interests.

\section{References}

1. Amaral LM, Cunningham MW Jr, Cornelius DC and LaMarca B: Preeclampsia: Long-term consequences for vascular health. Vasc Health Risk Manag 11: 403-415, 2015.

2. Sutton EF, Lob HE, Song J, Xia Y, Butler S, Liu CC, Redman LM and Sones JL: Adverse metabolic phenotype of female offspring exposed to preeclampsia in utero: A characterization of the $\mathrm{BPH} / 5$ mouse in postnatal life. Am J Physiol Regul Integr Comp Physiol 312: R485-R491, 2017.

3. Weissgerber TL and Mudd LM: Preeclampsia and diabetes. Curr Diab Rep 15: 9, 2015.

4. Cheng SB and Sharma S: Preeclampsia and health risks later in life: An immunological link. Semin Immunopathol 38: 699-708, 2016.

5. Possomato-Vieira JS and Khalil RA: Mechanisms of endothelial dysfunction in hypertensive pregnancy and preeclampsia. Adv Pharmacol 77: 361-431, 2016.

6. Zeisel SH: Epigenetic mechanisms for nutrition determinants of later health outcomes. Am J Clin Nutr 89: 1488S-1493S, 2009.

7. Reynolds CM, Gray C, Li M, Segovia SA and Vickers MH: Early life nutrition and energy balance disorders in offspring in later life. Nutrients 7: 8090-8111, 2015.

8. Myatt L, Redman CW, Staff AC, Hansson S, Wilson ML, Laivuori H, Poston L and Roberts JM; Global Pregnancy CoLaboratory: Strategy for standardization of preeclampsia research study design. Hypertension 63: 1293-1301, 2014.

9. Singh HJ: Pre-eclampsia: Is it all in the placenta? Malays J Med Sci 16: 7-15, 2009.

10. Levine RJ, Maynard SE, Qian C, Lim KH, England LJ, Yu KF, Schisterman EF, Thadhani R, Sachs BP, Epstein FH, et al: Circulating angiogenic factors and the risk of preeclampsia. $\mathrm{N}$ Engl J Med 350: 672-683, 2004.

11. Herraiz I, Llurba E, Verlohren S and Galindo A; Spanish Group for the Study of Angiogenic Markers in Preeclampsia: Update on the diagnosis and prognosis of preeclampsia with the aid of the sFlt-1/ PlGF ratio in singleton pregnancies. Fetal Diagn Ther 43: $81-89,2018$

12. Harmon AC, Cornelius DC, Amaral LM, Faulkner JL, Cunningham MW Jr, Wallace K and LaMarca B: The role of inflammation in the pathology of preeclampsia. Clin Sci (Lond) 130: 409-419, 2016.

13. Bouças AP, de Souza BM, Bauer AC and Crispim D: Role of innate immunity in preeclampsia: A systematic review. Reprod Sci 24: 1362-1370, 2017

14. Hashemi V, Dolati S, Hosseini A, Gharibi T, Danaii S and Yousefi M: Natural killer T cells in preeclampsia: An updated review. Biomed Pharmacother 95: 412-418, 2017. 
15. Mary S, Kulkarni MJ, Malakar D, Joshi SR, Mehendale SS and Giri AP: Placental proteomics provides insights into pathophysiology of pre-eclampsia and predicts possible markers in plasma. J Proteome Res 16: 1050-1060, 2017.

16. Myatt L and Cui X: Oxidative stress in the placenta. Histochem Cell Biol 122: 369-382, 2004.

17. Laresgoiti-Servitje E: A leading role for the immune system in the pathophysiology of preeclampsia. J Leukoc Biol 94: 247-257, 2013.

18. Robertson SA, Care AS and Moldenhauer LM: Regulatory $\mathrm{T}$ cells in embryo implantation and the immune response to pregnancy. J Clin Invest 128: 4224-4235, 2018.

19. Fisher SJ: Why is placentation abnormal in preeclampsia? Am J Obstet Gynecol 213 (Suppl): S115-S122, 2015.

20. Miko E, Szereday L, Barakonyi A, Jarkovich A, Varga P and Szekeres-Bartho J: Immunoactivation in preeclampsia: Vdelta2+ and regulatory $\mathrm{T}$ cells during the inflammatory stage of disease. J Reprod Immunol 80: 100-108, 2009.

21. Sharkey AM, Xiong S, Kennedy PR, Gardner L, Farrell LE, Chazara O, Ivarsson MA, Hiby SE, Colucci F and Moffett A: Tissue-Specific Education of Decidual NK Cells. J Immunol 195 3026-3032, 2015.

22. Kennedy PR, Chazara O, Gardner L, Ivarsson MA, Farrell LE, Xiong S, Hiby SE, Colucci F, Sharkey AM and Moffett A: Activating KIR2DS4 Is Expressed by Uterine NK Cells and Contributes to Successful Pregnancy. J Immunol 197: 4292-4300, 2016.

23. Xiong S, Sharkey AM, Kennedy PR, Gardner L, Farrell LE, Chazara O, Bauer J, Hiby SE, Colucci F and Moffett A: Materna uterine NK cell-activating receptor KIR2DS1 enhances placentation. J Clin Invest 123: 4264-4272, 2013.

24. Colonna M, Borsellino G, Falco M, Ferrara GB and Strominger JL: HLA-C is the inhibitory ligand that determines dominant resistance to lysis by NK1- and NK2-specific natural killer cells. Proc Natl Acad Sci USA 90: 12000-12004, 1993.

25. Zhang Y, Wang Y, Wang XH, Zhou WJ, Jin LP and Li MQ Crosstalk between human endometrial stromal cells and decidual NK cells promotes decidualization in vitro by upregulating IL 25. Mol Med Rep 17: 2869-2878, 2018.

26. Faas MM and de Vos P: Uterine NK cells and macrophages in pregnancy. Placenta 56: 44-52, 2017.

27. Moffett A and Colucci F: Uterine NK cells: Active regulators at the maternal-fetal interface. J Clin Invest 124: 1872-1879, 2014.

28. Lapaire O, Grill S, Lalevee S, Kolla V, Hösli I and Hahn S: Microarray screening for novel preeclampsia biomarker candidates. Fetal Diagn Ther 31: 147-153, 2012.

29. Nishizawa H, Pryor-Koishi K, Kato T, Kowa H, Kurahashi $H$ and Udagawa Y: Microarray analysis of differentially expressed fetal genes in placental tissue derived from early and late onset severe pre-eclampsia. Placenta 28: 487-497, 2007.

30. Leavey K, Bainbridge SA and Cox BJ: Large scale aggregate microarray analysis reveals three distinct molecular subclasses of human preeclampsia. PLoS One 10: e0116508, 2015.

31. Louwen F, Muschol-Steinmetz C, Reinhard J, Reitter A and Yuan J: A lesson for cancer research: Placental microarray gene analysis in preeclampsia. Oncotarget 3: 759-773, 2012.

32. Kaartokallio T, Cervera A, Kyllönen A, Laivuori K, Kere J and Laivuori H; FINNPEC Core Investigator Group: Gene expression profiling of pre-eclamptic placentae by RNA sequencing. Sci Rep 5: 14107, 2015.

33. Tong J, Zhao W, Lv H, Li WP, Chen ZJ and Zhang C: Transcriptomic Profiling in Human Decidua of Severe Preeclampsia Detected by RNA Sequencing. J Cell Biochem 119: 607-615, 2018

34. Kukurba KR and Montgomery SB: RNA Sequencing and analysis. Cold Spring Harb Protoc 2015: 951-969, 2015.

35. Kobayashi $\mathrm{H}$ : The impact of maternal-fetal genetic conflict situations on the pathogenesis of preeclampsia. Biochem Genet 53: 223-234, 2015

36. Kobayashi $\mathrm{H}$ : Characterization of the down-regulated genes identified in preeclampsia placenta. Hypertens Pregnancy 35: $15-21,2016$

37. Kleinrouweler CE, van Uitert M, Moerland PD, Ris-Stalpers C, van der Post JA and Afink GB: Differentially expressed genes in the pre-eclamptic placenta: A systematic review and meta-analysis. PLoS One 8: e68991, 2013.

38. Vaiman D, Calicchio R and Miralles F: Landscape of transcriptional deregulations in the preeclamptic placenta. PLoS One 8 e65498, 2013.
39. Masoura S, Kalogiannidis IA, Gitas G, Goutsioulis A, Koiou E Athanasiadis A and Vavatsi N: Biomarkers in pre-eclampsia: A novel approach to early detection of the disease. J Obstet Gynaecol 32: 609-616, 2012.

40. Garrido-Gomez T, Dominguez F, Quiñonero A, Diaz-Gimeno P, Kapidzic M, Gormley M, Ona K, Padilla-Iserte P, McMaster M, Genbacev O, et al: Defective decidualization during and after severe preeclampsia reveals a possible maternal contribution to the etiology. Proc Natl Acad Sci USA 114: E8468-E8477, 2017.

41. Conrad KP, Rabaglino MB and Post Uiterweer ED: Emerging role for dysregulated decidualization in the genesis of preeclampsia. Placenta 60: 119-129, 2017.

42. Luo S, Cao N, Tang Y and Gu W: Identification of key microRNAs and genes in preeclampsia by bioinformatics analysis. PLoS One 12: e0178549, 2017.

43. Tejera E, Cruz-Monteagudo M, Burgos G, Sánchez ME, Sánchez-Rodríguez A, Pérez-Castillo Y, Borges F, Cordeiro MNDS, Paz-Y-Miño C and Rebelo I: Consensus strategy in genes prioritization and combined bioinformatics analysis for preeclampsia pathogenesis. BMC Med Genomics 10: $50,2017$.

44. Yeung KR, Chiu CL, Pidsley R, Makris A, Hennessy A and Lind JM: DNA methylation profiles in preeclampsia and healthy control placentas. Am J Physiol Heart Circ Physiol 310: H1295-H1303, 2016.

45. Martin E, Ray PD, Smeester L, Grace MR, Boggess K and Fry RC: Epigenetics and preeclampsia: Defining functional epimutations in the preeclamptic placenta related to the TGF- $\beta$ pathway. PLoS One 10: e0141294, 2015.

46. Powers RW, Roberts JM, Plymire DA, Pucci D, Datwyler SA, Laird DM, Sogin DC, Jeyabalan A, Hubel CA and Gandley RE: Low placental growth factor across pregnancy identifies a subset of women with preterm preeclampsia: Type 1 versus type 2 preeclampsia? Hypertension 60: 239-246, 2012.

47. Jafri S and Ormiston ML: Immune regulation of systemic hypertension, pulmonary arterial hypertension, and preeclampsia: Shared disease mechanisms and translational opportunities. Am J Physiol Regul Integr Comp Physiol 313: R693-R705, 2017.

48. Rosenblum MD, Way SS and Abbas AK: Regulatory T cell memory. Nat Rev Immunol 16: 90-101, 2016.

49. Moffett A, Chazara O, Colucci F and Johnson MH: Variation of maternal KIR and fetal HLA-C genes in reproductive failure: Too early for clinical intervention. Reprod Biomed Online 33: 763-769, 2016.

50. Fu B, Tian Z and Wei H: Subsets of human natural killer cells and their regulatory effects. Immunology 141: 483-489, 2014

51. Moffett-King A: Natural killer cells and pregnancy. Nat Rev Immunol 2: 656-663, 2002

52. González A, Rebmann V, LeMaoult J, Horn PA, Carosella ED and Alegre E: The immunosuppressive molecule HLA-G and its clinical implications. Crit Rev Clin Lab Sci 49: 63-84, 2012.

53. Luo FY, Liu XH, Yang Y, He GL and Chen M: Expression of NKG2A and NKG2C receptors and their ligand HLA-E in decidua of preeclampsia patients. Sichuan Da Xue Xue Bao Yi Xue Ban 45: 582-586, 2014 (In Chinese).

54. Hiby SE, Apps R, Sharkey AM, Farrell LE, Gardner L, Mulder A, Claas FH, Walker JJ, Redman CW, Morgan L, et al: Maternal activating KIRs protect against human reproductive failure mediated by fetal HLA-C2. J Clin Invest 120: 4102-4110, 2010.

55. Kovats S, Main EK, Librach C, Stubblebine M, Fisher SJ and DeMars R: A class I antigen, HLA-G, expressed in human trophoblasts. Science 248: 220-223, 1990.

56. Chazara O, Xiong S and Moffett A: Maternal KIR and fetal HLA-C: A fine balance. J Leukoc Biol 90: 703-716, 2011.

57. Quach K, Grover SA, Kenigsberg S and Librach CL: A combination of single nucleotide polymorphisms in the 3'untranslated region of HLA-G is associated with preeclampsia. Hum Immunol 75: 1163-1170, 2014.

58. Rojas JM, Avia M, Martín V and Sevilla N: IL-10: A multifunctional cytokine in viral infections. J Immunol Res 2017: 6104054 , 2017.

59. Cheng SB and Sharma S: Interleukin-10: A pleiotropic regulator in pregnancy. Am J Reprod Immunol 73: 487-500, 2015.

60. Hennessy A, Pilmore HL, Simmons LA and Painter DM: A deficiency of placental IL-10 in preeclampsia. J Immunol 163: 3491-3495, 1999.

61. Tang Y, Liu H, Li H, Peng T, Gu W and Li X: Hypermethylation of the HLA-G promoter is associated with preeclampsia. Mol Hum Reprod 21: 736-744, 2015. 
62. Djurisic S and Hviid TV: HLA Class Ib Molecules and Immune Cells in Pregnancy and Preeclampsia. Front Immunol 5: 652, 2014.

63. Heinrichs $\mathrm{H}$ and Orr HT: HLA non-A,B,C class I genes: Their structure and expression. Immunol Res 9: 265-274, 1990.

64. Graff-Baker AN, Orozco JIJ, Marzese DM, Salomon MP, Hoon DSB and Goldfarb M: Epigenomic and transcriptomic characterization of secondary breast cancers. Ann Surg Oncol 25 : 3082-3087, 2018.

65. Hakam MS, Miranda-Sayago JM, Hayrabedyan S, Todorova K, Spencer PS, Jabeen A, Barnea ER and Fernandez N: Preimplantation factor (PIF) promotes HLA-G, -E, -F, -C expression in JEG-3 choriocarcinoma cells and endogenous progesterone activity. Cell Physiol Biochem 43: 2277-2296, 2017.

66. Uhrberg M, Valiante NM, Shum BP, Shilling HG Lienert-Weidenbach K, Corliss B, Tyan D, Lanier LL and Parham P: Human diversity in killer cell inhibitory receptor genes. Immunity 7: 753-763, 1997.

67. 67. Small HY, Akehurst C, Sharafetdinova L, McBride MW, McClure JD, Robinson SW, Carty DM, Freeman DJ and Delles C: HLA gene expression is altered in whole blood and placenta from women who later developed preeclampsia. Physiol Genomics 49: 193-200, 2017.

68. Hara N, Fujii T, Yamashita T, Kozuma S, Okai T and Taketani Y: Altered expression of human leukocyte antigen G (HLA-G) on extravillous trophoblasts in preeclampsia: Immunohistological demonstration with anti-HLA-G specific antibody ' $87 \mathrm{G}$ ' and anti-cytokeratin antibody 'CAM5.2'. Am J Reprod Immunol 36 : 349-358, 1996.

69. Yie SM, Li LH, Li YM and Librach C: HLA-G protein concentrations in maternal serum and placental tissue are decreased in preeclampsia. Am J Obstet Gynecol 191: 525-529, 2004.

70. Loisel DA, Billstrand C, Murray K, Patterson K, Chaiworapongsa T, Romero R and Ober C: The maternal HLA-G $1597 \Delta \mathrm{C}$ null mutation is associated with increased risk of pre-eclampsia and reduced HLA-G expression during pregnancy in African-American women. Mol Hum Reprod 19: 144-152, 2013.

71. López AS, Alegre E, LeMaoult J, Carosella E and González A: Regulatory role of tryptophan degradation pathway in HLA-G expression by human monocyte-derived dendritic cells. Mol Immunol 43: 2151-2160, 2006.

72. Lefebvre S, Berrih-Aknin S, Adrian F, Moreau P, Poea S, Gourand L, Dausset J, Carosella ED and Paul P: A specific interferon (IFN)-stimulated response element of the distal HLA-G promoter binds IFN-regulatory factor 1 and mediates enhancement of this nonclassical class I gene by IFN-beta. J Biol Chem 276: 6133-6139, 2001.

73. Guan Z, Song B, Liu F, Sun D, Wang K and Qu H: TGF- $\beta$ induces HLA-G expression through inhibiting miR-152 in gastric cancer cells. J Biomed Sci 22: 107, 2015.
74. Jabeen A, Miranda-Sayago JM, Obara B, Spencer PS, Dealtry GB, Hayrabedyan S, Shaikly V, Laissue PP and Fernández N: Quantified colocalization reveals heterotypic histocompatibility class I antigen associations on trophoblast cell membranes: Relevance for human pregnancy. Biol Reprod 89: 94, 2013.

75. Tilburgs T, Meissner TB, Ferreira LMR, Mulder A, Musunuru K, Ye $J$ and Strominger JL: NLRP2 is a suppressor of NF- $\kappa B$ signaling and HLA-C expression in human trophoblasts. Biol Reprod 96: 831-842, 2017.

76. Sun M, Song MM, Wei B, Gao Q, Li L, Yao B, Chen L, Lin L, Dai Q, Zhou X, et al: 5-Hydroxymethylcytosine-mediated alteration of transposon activity associated with the exposure to adverse in utero environments in human. Hum Mol Genet 25: 2208-2219, 2016.

77. Li X, Wu C, Shen Y, Wang K, Tang L, Zhou M, Yang M, Pan T, Liu $\mathrm{X}$ and $\mathrm{Xu} \mathrm{W}$ : Ten-eleven translocation 2 demethylates the MMP9 promoter, and its down-regulation in preeclampsia impairs trophoblast migration and invasion. J Biol Chem 293. 10059-10070, 2018.

78. Verloes A, Spits C, Vercammen M, Geens M, LeMaoult J, Sermon K, Coucke W and Van de Velde H: The role of methylation, DNA polymorphisms and microRNAs on HLA-G expression in human embryonic stem cells. Stem Cell Res (Amst) 19: 118-127, 2017.

79. Castelli EC, Veiga-Castelli LC, Yaghi L, Moreau P and Donadi EA: Transcriptional and posttranscriptional regulations of the HLA-G gene. J Immunol Res 2014: 734068, 2014.

80. van den Elsen PJ, van der Stoep N, Viëtor HE, Wilson L, van Zutphen M and Gobin SJ: Lack of CIITA expression is central to the absence of antigen presentation functions of trophoblast cells and is caused by methylation of the IFN-gamma inducible promoter (PIV) of CIITA. Hum Immunol 61: 850-862, 2000.

81. Ye Q, Shen Y, Wang X, Yang J, Miao F, Shen C and Zhang J: Hypermethylation of HLA class I gene is associated with HLA class I down-regulation in human gastric cancer. Tissue Antigens 75: 30-39, 2010.

82. McKelvey KJ, Ariyakumar G and McCracken SA: Inflammatory and Immune System Markers. Methods Mol Biol 1710: 85-101, 2018.

83. Martinez-Jimenez CP and Sandoval J: Epigenetic crosstalk: A molecular language in human metabolic disorders. Front Biosci (Schol Ed) 7: 46-57, 2015.

This work is licensed under a Creative Commons Attribution-NonCommercial-NoDerivatives 4.0 International (CC BY-NC-ND 4.0) License. 